\title{
Meiosis in the leek (Allium porrum L.) revisited. II. Metaphase I observations
}

\author{
G. H. JONES*, K. A. KHAZANEHDARI \& B. V. FORD-LLOYD \\ School of Biological Sciences, University of Birmingham, Birmingham B15 2TT, U.K.
}

\begin{abstract}
A detailed analysis of metaphase I of meiosis in pollen mother cells of Allium porrum has confirmed that despite frequent quadrivalent pairing (71 per cent) at prophase I and a high mean chiasma frequency ( 3.5 per tetrasome), the majority of quadrivalents are resolved into bivalent associations at metaphase I. The present observations also support the view that quadrivalent resolution results from the pronounced proximal chiasma localization found in this species. However, neither chiasma localization nor quadrivalent resolution are as prevalent as most previous reports have indicated. About 2 per cent of chiasmata, considerably more than in previous studies, occur in nonproximal chromosome regions in this material, while 12.9 per cent of metaphase I cells contained one or more multivalents, again exceeding most previous estimates. Meiotic regularity in this material was also disturbed by an unexpectedly high frequency of univalents. Overall 25.8 per cent of cells contained one or more univalent pairs, giving an overall mean of 0.32 univalent pairs per cell. It appears that meiosis in the leek is less regular than previous reports have indicated. The implications for fertility, stability and uniformity are not trivial and ought to be considered in the context of leek production and breeding.
\end{abstract}

Keywords: Allium porrum, chiasmata, meiosis, quadrivalents, tetraploid, univalents.

\section{Introduction}

A detailed reinvestigation of prophase I chromosome synapsis in the tetraploid leek, Allium porrum, $(2 n=4 \mathrm{x}=32)$ based on electron microscopy of surface-spread meiocytes has confirmed the earlier finding by Levan (1940) that quadrivalent synaptic associations occur frequently at this stage (Khazanehdari et al., 1995). The higher resolution of electron microscopy permitted the first detailed quantitative analysis of synaptic behaviour in $A$. porrum, which indicated a mean prophase I quadrivalent frequency of 5.68 per cell ( 71 per cent quadrivalents).

Meiosis in $A$. porrum, and in its close relative and possible wild ancestor $A$. ampeloprasum, is notable because despite their propensity for quadrivalent formation at prophase I, virtually all these quadrivalents are resolved into bivalents by metaphase I. Levan (1940) and subsequent investigators and commentators (e.g. Gohil \& Koul, 1977; Jones, 1990) realized that this remarkable behaviour is a consequence of the pronounced proximal localiza-

*Correspondence. tion of chiasmata in these species. Most chromosomes are associated by two very proximal chiasmata. As pairing partner switches (PPS) are unlikely to occur precisely between two proximal chiasmata, the main condition for quadrivalent retention is not met and, as a result, quadrivalents fall apart into two bivalents having characteristic cross shapes because of their very proximal chiasmata (Fig. 1).

Previous studies have observed that quadrivalents do occasionally persist into metaphase I, but this has been generally reported to be a quite rare occurrence (Levan, 1940; Koul \& Gohil, 1970; Gohil \& Koul, 1977; Wajahatulla, 1994) except for one smallscale study by Kadry \& Kamel (1955) who noted five quadrivalents in 26 metaphase I cells. As part of this current reinvestigation of meiosis in the leek, the EM analysis of prophase I synaptic associations has been followed up by a careful analysis of metaphase I chromosome associations. The previously described situation of almost universal bivalent formation at metaphase $\mathrm{I}$, with only rare instances of multivalent retention was not confirmed. Multivalents occurred much more frequently than previously recognized and, in addition, univalent pairs were 
Prophase I

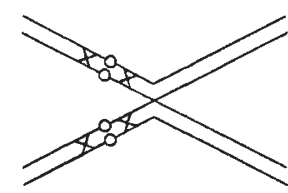

(b)

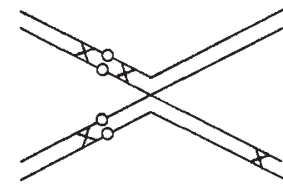

(c)

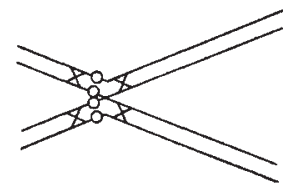

Metaphase I
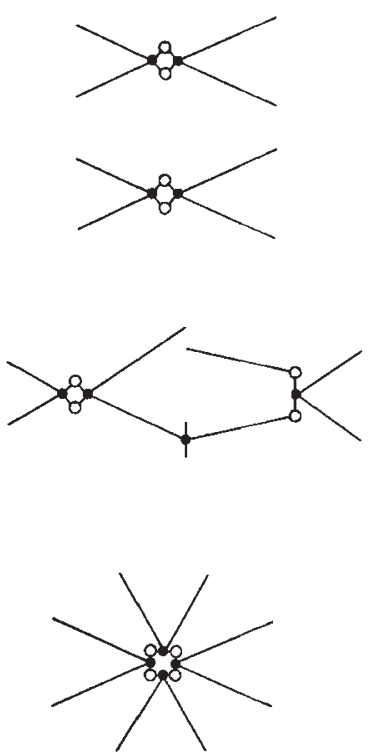

Fig. 1 The origins of different metaphase I configurations in Allium porrum, showing various prophase I situations and their metaphase I outcomes. In all cases shown a single PPS is present at prophase I. When all chiasmata are proximally localized ( $a$ and c) PPSs usually occur nonproximally leading to quadrivalent resolution and the appearance of bivalent pairs with proximally localized chiasmata at metaphase I(a); rarely a PPS may occur at or near to the centromere so that it is flanked by proximal chiasmata leading to star-shaped quadrivalent formation at metaphase I (c). Occasionally, nonproximal chiasmata occur, leading in some cases to chain quadrivalents (b). $O$ : centromeres; X: prophase I chiasmata; $\bullet$ : metaphase I chiasmata.

unexpectedly present in a proportion of cells. This paper also considers the conditions required for quadrivalent retention and the appearance of univalents at metaphase I. The implications of this pattern of meiotic association for fertility, stability and uniformity of leeks are discussed.

\section{Prophase I synapsis and metaphase I associations: theoretical considerations}

The general relationship between prophase I synaptic associations and metaphase I configurations in tetraploids is well known and has been discussed at length by earlier writers (Sybenga, 1975; Jones \& Vincent, 1994). For prophase I quadrivalent synaptic associations to persist to metaphase they must contain sufficient appropriately placed chiasmata. So, for example, in the simple situation of a quadrivalent having a single PPS, at least three chiasmata must be present and distributed in such a way that chiasmata occur on either side of the PPS. Given a widespread distribution of chiasma positions and PPS locations, there is a reasonably high probability that these conditions will be met, leading to quadrivalent maintenance at metaphase I. Quadrivalent retention is even more likely when chiasmata are distally localized, which is a common situation in many plant species.

The situation in the tetraploid leek is unusual because of its marked proximal chiasma localization. Usually the four chromosomes in a tetrasome are associated pairwise by chiasmata located immediately adjacent to and on either side of the median or submedian centromeres. This clustering of chiasmata makes it most unlikely that any PPS will be flanked on either side by chiasmata thus removing the chief condition for quadrivalent retention. As a consequence the quadrivalent will fall apart into two bivalents at metaphase I (Fig. 1a). Nevertheless, a PPS may, rarely, be located at the centromere region inbetween the sites of proximal chiasmata. This will generate a particular type of 'star-shaped' quadrivalent having eight chromosome arms radiating out from the centromerically clustered chiasmata and PPS (Fig. 1c).

Metaphase I quadrivalents, of different types, may also result when one or more nonproximal chiasmata are present. Although proximal chiasma localization is the rule in the leek, this control occasionally breaks down and one or more interstitial or distal chiasmata can form. When this happens, chain or even ring quadrivalents may appear at metaphase I because of the increased likelihood of PPSs in noncentromeric regions being flanked by chiasmata (Fig. 1b).

\section{Materials and methods}

The four cultivars of $A$. porrum included in this study were chosen to represent a range of cultivation ages (Khazanehdari et al., 1995). Musselburgh was selected as a representative of old-established varieties as it has been in cultivation for at least 110 years and probably much longer. Modern cultivars are represented by Profina and Verina while King Richard is regarded as a cultivar of intermediate age. For meiotic studies, plants were raised from seed sown in the spring and grown on in pots or in an experimental field where they were overwintered. Plants grown in this way flowered naturally the following spring or if grown in pots their flowering could be advanced by providing extra heat and light.

Metaphase I was analysed from acetic-orcein stained squash preparations of pollen mother cells. 
The best preparations were obtained from fresh unfixed anthers tapped out and squashed in acetic orcein stain, as prefixation of this material causes varying degrees of chromosome stickiness which hinders metaphase I analysis. A range of cytological fixatives was tested for their effects on chromosome stickiness, but none was entirely satisfactory. The best fixation was obtained with $1: 3$ propionic acidethanol, saturated with ferric acetate, at room temperature for 1 week followed by storage at $4{ }^{\circ} \mathrm{C}$ (H. de Jong, personal communication).

\section{Results and discussion}

Metaphase I chromosome configurations (quadrivalents, trivalents, bivalents, univalents) were counted in a minimum of 50 cells per plant from 15 plants belonging to four different leek varieties. Chiasma scoring required much clearer and better spread cells and was limited to a minimum of 15 cells per plant.

\section{Chiasma frequency and distribution}

The mean chiasma frequencies of individual plants ranged from 24.67 to 30.80 per cell, while the mean chiasma frequencies of the different varieties ranged from 27.03 to 28.78 with an overall mean of 28.07 chiasmata per cell, or 3.5 chiasmata per tetrasome (Table 1). Evidently the numbers of chiasmata formed are sufficient to maintain all the chromosomes as bivalents or quadrivalents, provided they occur in appropriate locations. The variation in chiasma frequency between varieties is not significant $\left(\mathrm{VR}_{3,11}<1\right)$ and is much less than the differences shown by individual plants within varieties.

Chiasma locations were recorded by visually classifying each chiasma into one of three zones along each chromosome arm, namely proximal, interstitial and distal, corresponding to equal subdivisions of each arm from the centromere to the telomere (Table 1). It is obvious that chiasmata show pronounced proximal localization because, overall, 98.1 per cent of chiasmata occurred in proximal regions and only 1.9 per cent occurred in nonproximal locations. The proportions of proximal and nonproximal chiasmata showed some variation between plants and also between different varieties. Nevertheless, the overall impression is of pronounced proximal localization in all plants and varieties analysed, with only occasional interstitial and distal chiasmata. Earlier reports by Levan (1940) and Koul \& Gohil (1970) also indicated that chiasma localization was incomplete, although the incidence of nonproximal chiasmata was considerably less in those studies than in the present study.

Table 1 Chiasma frequency and distribution in four varieties of leek

\begin{tabular}{|c|c|c|c|c|c|c|c|c|}
\hline \multirow[b]{2}{*}{ Variety } & \multirow[b]{2}{*}{ Plant } & \multirow[b]{2}{*}{ Cells } & \multicolumn{2}{|c|}{ Proximal chiasmata } & \multicolumn{3}{|c|}{ Nonproximal chiasmata } & \multirow{2}{*}{$\begin{array}{c}\text { Total } \\
\text { mean/cell }\end{array}$} \\
\hline & & & $N$ & Mean/cell & Interstitial & Distal & Mean/cell & \\
\hline \multirow[t]{4}{*}{ Verina } & 1 & 15 & 433 & 28.87 & 3 & 2 & 0.33 & 29.20 \\
\hline & 2 & 15 & 416 & 27.73 & 3 & 6 & 0.60 & 28.33 \\
\hline & 3 & 15 & 414 & 27.60 & 1 & 4 & 0.33 & 27.93 \\
\hline & 4 & 15 & 443 & 29.53 & & 2 & 0.13 & 29.67 \\
\hline Mean & & & & 28.43 & 0.12 & 0.23 & 0.35 & 28.78 \\
\hline \multirow[t]{4}{*}{ Profina } & 1 & 15 & 375 & 25.00 & 1 & 5 & 0.40 & 25.40 \\
\hline & 2 & 17 & 495 & 29.12 & 4 & 8 & 0.71 & 29.82 \\
\hline & 3 & 15 & 358 & 23.87 & 3 & 9 & 0.80 & 24.67 \\
\hline & 4 & 16 & 440 & 27.50 & 1 & 4 & 0.31 & 27.81 \\
\hline Mean & & & & 26.48 & 0.14 & 0.41 & 0.56 & 27.03 \\
\hline \multirow{4}{*}{ King Richard } & 1 & 15 & 427 & 28.47 & 17 & 18 & 2.33 & 30.80 \\
\hline & 2 & 15 & 420 & 28.00 & 1 & 9 & 0.67 & 28.67 \\
\hline & 3 & 15 & 402 & 26.80 & 1 & & 0.07 & 26.87 \\
\hline & 4 & 15 & 386 & 25.73 & 2 & 6 & 0.53 & 26.27 \\
\hline Mean & & & & 27.25 & 0.35 & 0.55 & 0.90 & 28.15 \\
\hline \multirow{3}{*}{ Musselburgh } & 1 & 16 & 449 & 28.06 & & 1 & 0.06 & 28.13 \\
\hline & 2 & 15 & 421 & 28.07 & & 5 & 0.33 & 28.40 \\
\hline & 3 & 15 & 420 & 28.00 & 2 & 4 & 0.40 & 28.40 \\
\hline Mean & & & & 28.04 & 0.04 & 0.22 & 0.26 & 28.30 \\
\hline
\end{tabular}


Levan (1940) reported only three instances of nonproximal chiasmata in 250 cells at metaphase I, which is many fewer than found in the present study even allowing for possible differences in the criteria used for defining proximal versus nonproximal chiasmata.

\section{Metaphase / configuration frequencies}

As expected, bivalents were the predominant chromosome configuration at metaphase I (Fig. 2 and Table 2). The overall mean bivalent frequency was 15.43 per cell, which means that 96.4 per cent of all chromosomes were associated as bivalents at this stage with relatively little variation between plants or varieties. So, despite the preponderance of multivalents at prophase I, relatively few of them persisted to metaphase I. However, the frequency of metaphase I multivalents in this material, although much reduced compared with prophase $\mathrm{I}$, is not trivial and considerably exceeds most earlier reported frequencies for the leek. Most metaphase I nuclei contained no multivalents at all, but 12.92 per cent of cells contained either one or two multivalents (Fig. 2). The mean frequency of multivalents, overall, was 0.13 per cell of which virtually all were quadrivalents. In fact, only two instances of metaphase I trivalents were encountered among all the cells analysed. The majority of metaphase I multivalents (78 per cent) were chain or ring configurations, dependent on one or more interstitial or distal chiasmata for their retention. Only 22 per cent of multivalents were of the star-shaped type involving proximal chiasmata and centromerically located PPSs (Figs 1c and 2c). This again contrasts with Levan's (1940) study in which he reported a much lower quadrivalent frequency, with only 2.6 per cent of cells at metaphase I containing quadrivalents, compared with 12.92 per cent of cells in the present study. Furthermore, the majority of quadrivalents in Levan's study were of the star-shaped type whereas these were relatively infrequent in the present study. These differences and other differences in the chiasma data, indicate that Levan's material probably differed quite markedly from that analysed now, with regard to the proportion of nonproximal chiasmata, the incidence of multivalents at metaphase I and the proportions of quadrivalent types. This is not entirely surprising as the four varieties included in the present study show some variation for all

Table 2 Frequencies of metaphase I configurations in four varieties of leek

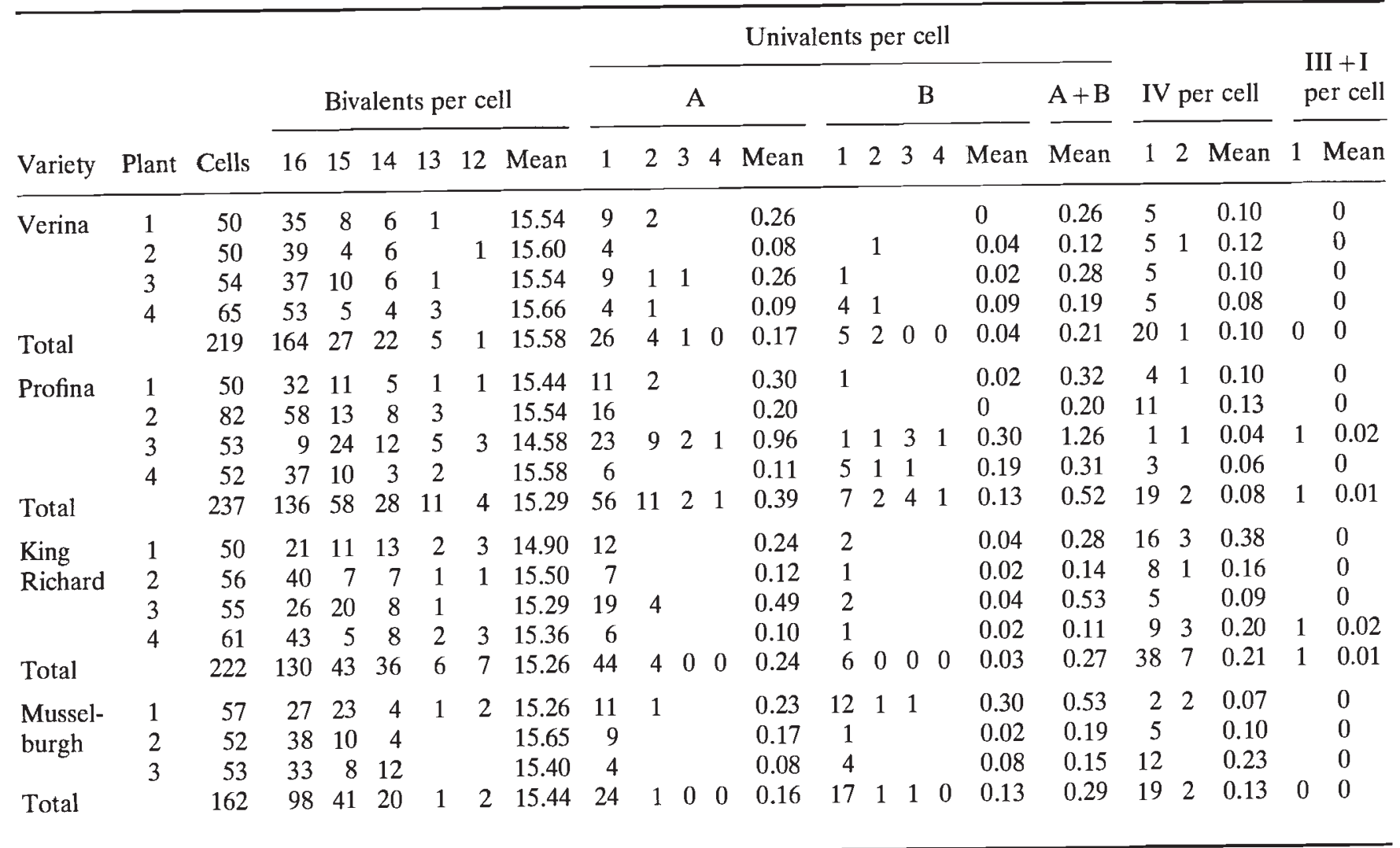

(c) The Genetical Society of Great Britain, Heredity, 76, 186-191. 
these variables. Unfortunately, the leek varieties analysed by Levan are not recorded. A small-scale study by Kadry \& Kamel (1955) provides the only previous indication of more frequent quadrivalent retention. They reported five quadrivalents in 26 metaphase I cells of $A$. porrum 'Giant Musselburgh' but the low number of cells analysed reduced the impact of this observation.

An unexpected feature of the metaphase I cells was a surprisingly frequently occurrence of univalent pairs, which were of two distinct morphological types (termed A and B). Type A univalents showed the classical univalent phenotype and occurred as separate and unconnected univalents occupying random locations in the cell (Fig. 2a). The other type (B), while being clearly achiasmate remained loosely associated by 'sticky' connections. Although both univalent types were observed in all plants, type A were considerably more frequent (Table 2).

Although the majority of nuclei in all varieties contained no univalents, the percentage of nuclei containing at least one pair of univalents was surprisingly high, ranging from 17.35 per cent to 35.02 per cent in different varieties with an overall mean of 25.81 per cent of nuclei. Because some cells contain two or more (up to four) pairs of univalents, these percentages under-represent the univalent frequencies. The overall mean frequency of univalents was 0.32 per cell (range 0.21-0.52 in different varieties). However, the four varieties analysed did not differ significantly as regards univalent frequency $\left(\mathrm{VR}_{3,11}<1\right)$ which emphasizes that this is a general

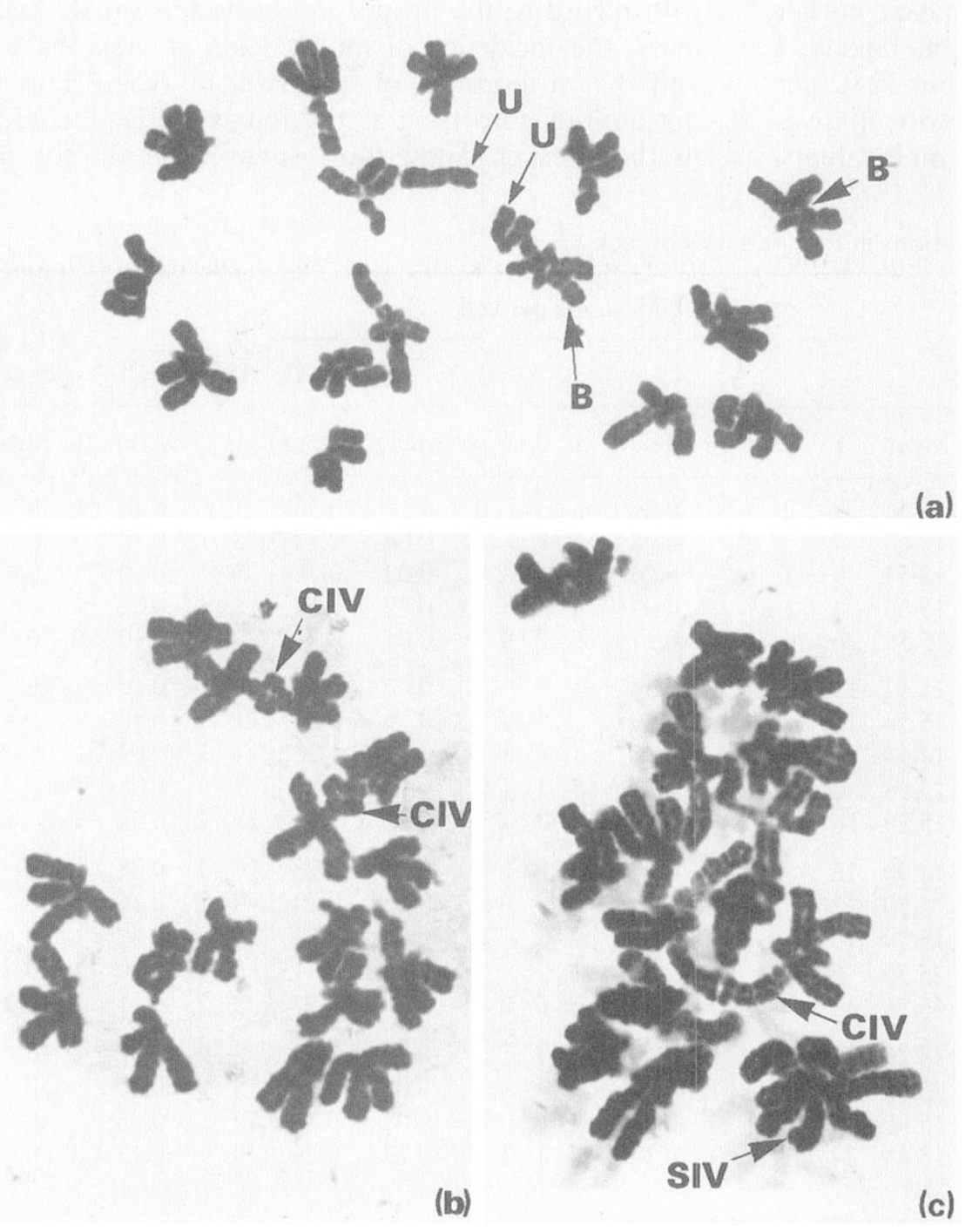

Fig. 2 Metaphase I configurations in Allium porrum. (a) Shows a cell with 15 bivalents and a single pair of univalents (U); the bivalents (B) are typically cruciform with pronounced proximal localization of chiasmata. (b and c) Examples of chain quadrivalents (CIV) and a star quadrivalent (SIV) which has eight arms radiating outwards from a central core (see Fig. 1c). $\times 1600$. 
feature of all the material examined in this study. Surprisingly, univalents were not reported, or only very infrequently, in earlier studies (e.g. Levan, 1940; Koul \& Gohil, 1970; Wajahatullah, 1994) apart from the study by Kadry \& Kamel (1955) which reported three univalent pairs among 26 cells.

Synapsis was generally complete or nearly so in all the prophase nuclei examined in the course of this study and consequently metaphase I univalents cannot be attributed to pairing failure (asynapsis). The relatively high mean chiasma frequency observed in this material is adequate to maintain all chromosomes as bivalents or multivalents, provided they are appropriately distributed among the paired regions. The appearance of univalents at metaphase I therefore must reflect inappropriate chiasma distributions such that, despite the relatively high mean chiasma frequency, some synapsed chromosome pairs remain achiasmate. Meiotic irregularity, in the form of univalents or quadrivalents, is not apparently caused by environmental stress which might result from conditions of additional heat and light applied to pot-grown plants as there was no discernible difference between pot-grown plants, whether or not exposed to extra heat and light, and field-grown plants which flowered naturally in the open air.

It has been argued that the meiotic system of the leek, involving a high degree of proximal chiasma localization, ensures almost invariable bivalent formation at metaphase I which in turn results in regular chromosome disjunction and the production of euploid balanced chromosome sets (Levan, 1940; Koul \& Gohil, 1970; Gohil 1984). The leek is therefore regarded and frequently cited as an example of a tetraploid which enjoys a high level of fertility and stability as a consequence of its unusual meiotic behaviour. The present study requires a reassessment of this view. All four leek varieties studied revealed unexpectedly high frequencies of multivalents and univalents at metaphase I, despite the overall preponderance of bivalents. Univalents are notoriously irregular in their disjunctional behaviour at anaphase I and anaphase II, and their presence is very likely to cause the production of aneuploid meiotic products and aneuploid zygotes. Although quadrivalents are less likely to cause disjunctional problems, as they can disjoin $2: 2$, it is known from other systems that they do not always behave so obligingly and 3:1 nondisjunctional separation can also result (Sybenga, 1975). The simple observation that 40 per cent of metaphase I cells in the leek contain univalents or multivalents, or both, raises serious doubts concerning the previous assumptions about its fertility and stability. The postmeiotic consequences of meiotic irregularity and the incidence of aneuploidy in the seedling population will be examined in a separate paper, together with an assessment of the implications of these findings for leek production and breeding.

\section{Acknowledgements}

We thank Mr B. Smith of Horticulture Research International, Wellesbourne, UK for his advice and stimulating discussions concerning leek genetics and breeding. One of us (K.K.) acknowledges receipt of an Agriculture and Food Research Council Postgraduate Studentship. Figure 2a was provided by kind permission of $\mathrm{Mr} \mathrm{M}$. K. Wajahatulla who undertook a preliminary study of leek meiosis as part of his MSc studies in this School.

\section{References}

GOHIL, R. N. 1984. Extent of recombination possible in the cultivated leek. Eucarpia 3rd Allium Symposium, Institute for Horticultural Plant Breeding (IVT), Wageningen, The Netherlands, 4-6 September 1984, pp. 99-105.

GOHIL, R. N. AND KOUL, A. K. 1977. The cause of multivalent suppression in Allium ampeloprasum L. Beitr. Biol. Pflanzen, 53, 473-478.

JONES, R. N. 1990. Cytogenetics. In: Rabinsowitch, H. D. and Brewster, J. C. (eds), Onions and Allied Crops, vol. 1, Botany, Physiology and Genetics, pp. 199-214. CRC Press, Boca Raton, FL.

JONES, G. H. AND VINCENT, J. E. 1994. Meiosis in autopolyploid Crepis capillaris. II. Autotetraploids. Genome, 37, 497-505.

KADRY, A. E. R. AND KAMEL, S. A. 1955. Cytological studies in the two tetraploid species of Allium kurrat Schureint and Allium porrum L. and their hybrid. Svensk. Bot Tidskr., 49, 314-324.

KHAZANEHDARI, K. A., JONES, G. H. AND FORD-LLOYD, B. V. 1995. Meiosis in the leek (Allium porrum L.) revisited. I. Prophase I pairing. Chromosome Res., 3, 433-439.

KOUL, A. K. AND GOHIL, R. N. 1970. Cytology of the tetraploid Allium ampeloprasum with chiasma localization. Chromosoma, 29, 12-19.

LeVAn, A. 1940. Meiosis of Allium porrum, a tetraploid species with chiasma localization. Hereditas, 26, $454-462$.

SYBENGA, J. 1975. Meiotic Configurations. Springer, Berlin. WAJAhATULlaH, M. K. 1994. The behaviour of meiotic chromosomes in leek, A. porrum L. Cytologia, 59, 333-338. 